\title{
LA CONSEJERÍA COMO INTERVENCIÓN DE SALUD MENTAL EN TRABAJADORES INDUSTRIALES.
}

\author{
COUNSELING LIKE MENTAL HEALTH INTERVENTION ON INDUSTRIAL WORKERS. \\ Sánchez-Armass Cappello Omar ${ }^{1}$, Purata Juárez Bella Dulce ${ }^{3}$, Galván Almazán Gicela de Jesús², Aradillas \\ García Celia².
}

Universidad Autónoma de San Luis Potosí: 1 Facultad de Psicología, 2 Facultad de Medicina/CIACYT. 3 Instituto de Estudios Superiores para el Desarrollo Integral, Campus SLP. México.

\section{RESUMEN}

Introducción: La Organización Mundial de la Salud estima que los trastornos mentales son la primera causa de discapacidad y la segunda de enfermedad en el mundo para personas entre 15-44 años. Objetivo: Fue observar los valores de autoestima, depresión, y ansiedad antes, y después de una intervención con consejería. Material y Método: Estudio longitudinal, descriptivo y observacional. Completaron el estudio 12 trabajadores de una empresa de la ciudad de San Luis Potosí, se les aplicaron las pruebas psicológicas, Rosemberg, CESD, y STAI antes y después de la intervención de consejería con de un mínimo de 8 sesiones durante un periodo de 10 meses. Resultados: Después de la intervención de consejería, se observaron un aumento significativo en la autoestima, y una disminución moderada de los síntomas de depresión y ansiedad, aunque sin significancia estadística. Conclusiones: Es posible que el buen resultado con autoestima este coadyuvado por la percepción de los trabajadores de ser gratificados al participar en el estudio. La no significancia en depresión y ansiedad, pueden ser debidas al pequeño tamaño de la muestra ya que se puede observar disminución en los síntomas para estas variables.

Palabras Clave: Trabajador, Consejería, Autoestima, Depresión, Ansiedad.

\section{ABSTRACT}

Introduction: The World Heat Organization states that mental disorders are the principal cause of disability and the second illness worldwide for persons between 15 to 44 years old. Objective: To observe self-esteem, depression, and anxiety values before personal counseling and after it. At least 8 sessions. Material and method: Longitudinal, descriptive, and observational study. 12 workers of an enterprise in San Luis Potosi finished the study. Psychological tests such as Rosemberg, CESD, and STAI were applied before and after the intervention. Results: A rise in self-esteem was observed, with statistical significance, but not at depression. Anxiety did not show changes. Conclusions: The results could be influenced by the workers' perception of being gratified for participating in the study. Our results may be affected by the small size of workers who finished the study.

Key words: Worker, Counselling, self-esteem, depression, anxiety.

Correspondencia: Celia Aradillas García celia@uaslp.mx

Recibido: 08 de julio 2021, aceptado: 19 de noviembre 2021

(C) Autor2022

\section{(c) (1)}

DOI: https://doi.org/10.29105/respyn21.1-1

Citation: Sánchez-Armass Cappello O., Purata Juárez B.D., Galván Almazán G.J., Aradillas García C. (2022) La consejería como intervención de salud mental en trabajadores industriales. Revista Salud

Pública y Nutrición, 21 (1), 1-9. 


\section{Introducción}

La Organización Mundial de la Salud (OMS) estima que los trastornos mentales son la primera causa de discapacidad y la segunda de enfermedad en el mundo para personas entre 15 a 44 años de edad (Asensio-Cuesta, Bresó, Saez, \& García-Gómez, 2019). Lo cual corresponden a la etapa de la vida en que se espera la mayor productividad en todas las esferas que conforman la vida de los seres humanos.

Durante los últimos años múltiples estudios han confirmado que el tratamiento de la depresión y la ansiedad son convenientes tanto para la salud, el bienestar como para los aspectos económicos relativos a la productividad; ya que es más rentable para la sociedad y el gobierno dar diagnóstico y tratamiento en tiempo y forma a estos padecimientos incapacitantes (Sporinova et al., 2019). Se estima que para el año 2030 el tratamiento de estos trastornos que conllevan enfermedad y pérdidas económicas, necesitarían una inversión de US $\$ 147$ billones lo que se traduciría en 43 millones de años de vida saludable y productiva con un valor de US $\$ 310$ billones, esto solo considerando los beneficios económicos, lo que hace una razón costo beneficio de 3:1(Chisholm et al., 2016). En un estudio realizado en nuestro país se mostró que las estrategias que dan un mejor costoefectividad son las que combinan manejo proactivo con psicoterapia breve y fármacos, esto calculado a partir de los años de vida ajustados por discapacidad (AVISA) que corresponde a la suma de los años perdidos por mortalidad prematura y los años vividos con discapacidad (Creswell et al., 2017; Lara-Muñoz et al., 2010).

Debido al alto costo de las intervenciones terapéuticas para tratar estos padecimientos surge importantemente el trabajo de consejería. $\mathrm{La}$ consejería es un tipo de coaching de vida enfocado en problemas interpersonales. Ésta incluye intervenciones tanto individuales como grupales, ayudando en la búsqueda de alternativas o soluciones a las personas que consultan, pero con la particularidad de ofrecer un servicio más a corto plazo que la psicoterapia (Bower, Knowles, Coventry, Rowland, \& Group, 2011).

Por otro lado, una de las principales metas del Programa Mundial de Acción en Salud Mental de la OMS es disminuir la carga de los trastornos mentales y promover acciones que permitan que la atención a estos desórdenes mentales esté al alcance de todos los seres humanos en todas las regiones del mundo, por lo que se hacen esfuerzos en el sentido de visibilizar estos problemas. Sin embargo, a pesar de todos los planes de salud mental, programas y leyes, las metas propuestas entre 2014 y 2020; no fueron alcanzadas. Habrá que mencionar y sumar, el impacto en los temas de salud mental que causo la epidemia por SARS-CoV-2, con la COVID-19 (Santomauro et al., 2021).

El trastorno depresivo (TD) es un problema de salud mental pública que se observa significativamente distribuido en todas las regiones del mundo, desde 2011 se encontró como la cuarta causa de discapacidad y la segunda en el 2020 (Evans-Lacko et al., 2018). El TD se encuentra asociado a factores de riesgo no modificables como condiciones individuales de edad y sexo, factores hereditarios, raza y etnia (Labaka, Goñi-Balentziaga, Lebeña, \& Pérez-Tejada, 2018; Ware et al., 2015). Así mismo ha mostrado correlación con factores de riesgo modificables que incluyen los aspectos socioculturales, el estilo de vida (alimentación, sedentarismo, vivienda, uso del tiempo libre, etc.), los entornos familiar y laboral, así como las variables macro y micro económicas de las diferentes regiones geográficas (Porras-Segovia et al., 2019; Sgroi, 2010). Por otro lado, la ansiedad es el trastorno psicológico más común, presente en casi todos los grupos culturales y puede ser experimentado alguna vez en la vida por el $32 \%$ de los seres humanos (Chisholm et al., 2016), que pese a ser una condición subjetiva y relativa es contemplada en la definición de salud mental de la OMS. Se ha reportado que cierto nivel de ansiedad interfiere con el desarrollo de las actividades diarias como los estudios, el trabajo, la interacción con otras personas; lo cual repercute el desarrollo personal, y la productividad. De este modo tenemos que una variable macro nivel como el crecimiento económico puede ser afectada por una variable micro nivel como la productividad a su vez relacionada con estados afectivos como la felicidad (Bower et al., 2011; Sgroi, 2010).

La autoestima es un componente psicológico que nos indica los sentimientos de valoración y respeto hacia la propia persona. Esta se va formando y desarrollando al tiempo en que el individuo interactúa con su ambiente e internaliza las experiencias físicas, psicológicas y sociales 
(Wilhelm, Martin, \& Miranda, 2012). Es conocido que una baja autoestima está relacionada con depresión y que esta última también puede incidir en la autoestima, estas relaciones parecen también observarse con ansiedad. En 2012 se llevó a cabo un meta análisis para dilucidarlas, se encontró que el efecto de autoestima sobre depresión fue mayor que el de depresión sobre autoestima; con ansiedad los resultados fueron similares, y no se encontraron diferencias significativas por sexo y edad (Sowislo \& Orth, 2013). Lo que sugiere que las intervenciones para aumentar la autoestima llevarían a una disminución en el riesgo de depresión. Por lo que resulta relevante estudiar este tema en especial en trabajadores industriales ya que en el ánimo de incrementar la productividad se está dando importancia al bienestar del trabajador (Hoffmeister, Gibbons, Schwatka, \& Rosecrance, 2015). También se ha encontrado que la autoestima muestra una relación positiva con el bienestar (Moreno \& Marrero, 2015).

Se ha reportado que una disminución del $5 \%$ en riesgos al bienestar del trabajador está significativamente asociada con una disminución en ausentismo del $0.74 \%$, una disminución de presentismo del $2.38 \%$, y el $0.24 \%$ de aumento del rendimiento en la actividad laboral (Shi, Sears, Coberley, \& Pope, 2013). Según reportó Hemp (2004) para el Harvard Business Review, el presentismo les costó a las empresas norteamericanas alrededor de $\$ 150$ billones de dólares al año y se entiende como el hecho de asistir a trabajar sintiéndose mal, sin ánimo y con un bajo desempeño, lo que causa un impacto mayor que el ausentismo (Adrián Lazo Páez, 2015). Entre las condiciones de salud más frecuentes se encuentran los trastornos del ánimo, y afecciones físicas crónicas (Bielecky et al., 2015).

Cabe mencionar que en nuestro país la Ley Federal del Trabajo, artículo 132, fracción XVI, obliga al patrón a instalar fábricas, talleres, oficinas, locales y demás lugares donde deban desempeñarse los trabajadores, de acuerdo al reglamento y las normas oficiales mexicanas en materia de seguridad, salud y medio ambiente de trabajo, para prevenir accidentes y enfermedades laborales. Esta misma ley dispone en su artículo 512 "que en los reglamentos e instructivos que las autoridades laborales expidan, se fijen las medidas necesarias para prevenir los riesgos de trabajo y lograr que el trabajo se realice en condiciones que aseguren la vida y la salud de los trabajadores" (Secretaría del Trabajo y Previsión Social, 2014).

Por otro lado, se muestra que el concepto salud solo se encuentra mencionado de manera general. Recientemente, El Diario Oficial de la Federación, publico el 23 de octubre de 2018 que el Comité Consultivo Nacional de Normalización de Seguridad y Salud en el Trabajo, en su Primera Sesión Ordinaria de 2017, otorgó la aprobación respectiva a la NOM 35, Norma Oficial Mexicana que garantiza por ley la identificación y análisis de riesgo psicosocial en el trabajo. Se da visibilidad a la necesidad de realizar más estudios de este tipo a pesar de ser difíciles y costosos de llevar a cabo.

El estado de San Luis Potosí cuenta con una planta de trabajadores afiliados al Instituto Mexicano del Seguro Social hasta antes de la pandemia, en febrero del año 2020, de 455 mil 287 empleos formales de acuerdo con la Secretaría del Trabajo y Previsión Social, Subsecretaría de Empleo y Productividad Laboral. Estas estadísticas solo consideran como riesgos de trabajo los accidentes de trabajo propiamente dichos, solamente 2016 fueron $(7,388)$, los accidentes en trayecto al centro laboral $(4,078)$, y las enfermedades laborales (270). No se ha localizado más información local lo que sugiere que no existen estudios debido a que no se le ha dado la importancia seguramente por desconocimiento de la conveniencia de cuidar la salud y bienestar del trabajador (Secretaría del Trabajo y Previsión Social, 2014).

Los factores de riesgo para la salud física y mental y en general el bienestar de los trabajadores se derivan de modo en que la organización estructura sus tareas y responsabilidades, por lo que es necesario intervenir directamente en el nivel organizacional para prevenir y afrontar estos problemas.

El objetivo de este estudio fue observar el comportamiento de las mediciones de autoestima, depresión y ansiedad antes y después de la intervención de consejería y la relación que guardan entre sí. 


\section{Material y Método}

Es un estudio piloto, longitudinal descriptivo y observacional. El presente trabajo se apega a lo señalado por la declaración de Helsinki ("World Medical Association Declaration of Helsinki: ethical principles for medical research involving human subjects", 2013) y lo dispuesto en la Ley general de Salud en materia de investigación para la salud. Posterior a detallar la información acerca de la naturaleza, propósito y condiciones de la investigación, así como las características, duración, anonimato de los datos recabados y el carácter voluntario de su participación, todos los participantes firmaron el consentimiento informado.

Se invitaron a participar en el estudio a 40 trabajadores que laboraban en una empresa de instalaciones eléctricas localizada en la zona industrial de la ciudad de San Luis Potosí. La participación fue, voluntaria, sin costo para los participantes, confidencial, y se les aclaró que podrían abandonar el estudio cuando así lo desearan. Los trabajadores tenían entre 23 y 46 años, y en su mayoría un nivel de educación media (secundaria técnica). Se aplicaron las pruebas psicológicas para medir autoestima, depresión, ansiedad; Rosemberg, CESD, y STAI respectivamente antes de la intervención de consejería y una vez más al terminó de la intervención. El estudio se realizó durante un periodo de 10 meses y solamente se tomaron en cuenta para el estudio, aquellos trabajadores que completaron entre 8 y 10 sesiones de consejería y que completaron las pruebas psicológicas antes, y después de la intervención, en total el universo de trabajo fue de 12 trabajadores. Habida cuenta de que se suspendieron actividades en días feriados, periodos vacacionales, y compromisos laborales de los trabajadores. También hubo trabajadores que se dieron de baja en el centro laboral. Los criterios de inclusión fueron: Estar dados de alta laboral en la empresa seleccionada y firmar una carta de consentimiento informado. Los criterios de eliminación fueron: no completar por lo menos 8 sesiones de consejería y las pruebas psicológicas.

Se eligió la prueba de Rosemberg que muestra los sentimientos de autovaloración y respeto de sí mismo, consta de 10 reactivos, 5 formulados en sentido negativo y 5 en positivo. Se interpreta como sigue: de 30 a 40 puntos autoestima elevada, deseable considerada normal. De 26 a 29 puntos media, no presenta problemas, aunque se aconseja mejorarla. Y menor de 25 puntos baja con problemas significativos, se recomienda atención especializada. La escala Rosemberg ha sido traducida y validada en español. Los valores para la consistencia interna de las escalas son 0.76 y 0.87 . La fiabilidad de 0.80(Rosenberg, 2016).

Para medir de depresión se usó la escala del Centro de Estudios Epidemiológicos de la Depresión (CESD-10), que es una versión corta muy usada en hombres y mujeres de diferentes edades en varios países. Consta de 10 reactivos que detectan síntomas afectivos como tristeza y bajo estado de ánimo y síntomas físicos como poca energía y problemas de sueño. De 0 a 14 puntos los síntomas no son consistentes con riesgo de depresión, la presencia de este trastorno es improbable. De 15 a 24 puntos existen síntomas consistentes con riesgo de depresión, la presencia de este trastorno es probable, se recomienda una evaluación diagnóstica formal. De 25 a 40 puntos los síntomas son muy consistentes con los criterios para riesgo de depresión, la presencia de este trastorno es muy probable, es necesaria una atención profesional especializada (ST, AC, \& HH, 2006).

Para evaluar los niveles de ansiedad se usó una versión corta de State Trait Anxiety Inventory (STAI) que consta de 10 reactivos, esta prueba está estandarizada para un nivel de lectura de sexto grado de educación básica en 48 idiomas e investiga síntomas como sentirse nervioso, preocupado, aumento de la frecuencia cardiaca, sudoración, dificultad para respirar asociados al trastorno de ansiedad. Se interpreta así: de 0 a 14 puntos el riesgo de un trastorno de ansiedad es improbable, no se recomienda una evaluación diagnóstica. De 15 a 24 puntos es probable el riesgo de un trastorno de ansiedad, se recomienda una evaluación diagnóstica formal. De 25 a 40 puntos es necesaria una evaluación profesional especializada (A, JB, \& RL, 2009).

Las pruebas psicológicas y las sesiones de consejería fueron aplicadas por 6 maestros en consejería. Los consejeros recibieron un pago en efectivo, de acuerdo con el número de sesiones de consejería que se llevaron a cabo, este pago corrió a cargo de la empresa donde laboraban los trabajadores. Las sesiones de consejería fueron semanales, con 
duración de 45 minutos, y el número de estas dependió de cada caso particular. También se realizó una sesión grupal de cierre que incluía un cuestionario de satisfacción auto administrado.

Se realizó la prueba estadística de análisis de fiabilidad en el programa estadístico SPSS para evaluar la confiabilidad de los instrumentos utilizados, a través del índice Alpha de Cronbach. Para la prueba del Centro de Estudios Epidemiológicos de la Depresión (CESD-10) se obtuvo un Alpha de .871, mientras que para la prueba State Trait Anxiety Inventory (STAI) versión corta el valor de Alpha fue de .876. Por lo tanto, fue adecuado utilizar la totalidad de ítems para cada una de las pruebas.

Se realizó una base de datos con las puntuaciones de cada uno de los instrumentos aplicados a los participantes, utilizando los programas de análisis estadístico SPSS versión 20.0 y GraphPad Prism versión 5. Se evaluó la normalidad de los datos mediante la prueba de normalidad Shapiro-Wilk y posteriormente se compararon medias con la prueba paramétrica $\mathrm{T}$ para muestras relacionadas. Por último, para evaluar la correlación entre la puntuación de los síntomas de autoestima, depresión y ansiedad se realizó una correlación de Pearson. Todos los valores se representan como media \pm la desviación estándar Valores de $\quad \mathrm{P}<0.05$ se consideraron como estadísticamente significativos.

\section{Resultados}

Se incluyeron para el análisis los datos de participantes que concluyeron un ciclo de 8 a 10 sesiones de consejería. Por lo anterior, se obtuvo una muestra de 12 trabajadores; 2 mujeres de 25 y 32 años respectivamente y 10 hombres de entre 23 y 44 años. En la Tabla 1 se muestra la puntuación para depresión, ansiedad y autoestima; antes y después del estudio.

En la Figura 1 podemos observar en las barras de color naranja un aumento en el puntaje de autoestima después de la consejería, cabe destacar que el único sujeto en el que no se observó una mejoría fue el folio 35. Además, la media del puntaje de autoestima de los participantes posterior a la consejería aumento de 24 a 31 puntos donde encontramos una diferencia significativa con una $\mathrm{t}=-3.350$, y significancia bilateral $=0.006$ (Tabla 2$)$. Esto es relevante ya que, de acuerdo con los puntos de corte de la prueba de Rossemberg, se considera una autoestima alta deseable una puntuación de 30 a 40. En relación con depresión y ansiedad no se encontraron diferencias significativas para los puntajes de los participantes del estudio, podemos observar en la Figura 2. Una tendencia en disminución del puntaje de la prueba consistente con una mejora del estado depresivo. Sin embargo, no alcanzó significancia estadística, de la misma manera no se encontraron diferencias significativas para el puntaje de ansiedad (Tabla 2).

\begin{tabular}{|c|c|c|c|c|c|c|c|c|}
\hline Folio & Edad & Sexo & $\begin{array}{c}\text { Depresión } \\
\text { antes }\end{array}$ & $\begin{array}{c}\text { Depresión } \\
\text { después }\end{array}$ & $\begin{array}{c}\text { Ansiedad } \\
\text { antes }\end{array}$ & $\begin{array}{c}\text { Ansiedad } \\
\text { después }\end{array}$ & $\begin{array}{c}\text { Autoestima } \\
\text { antes }\end{array}$ & $\begin{array}{l}\text { Autoestim } \\
\text { a después }\end{array}$ \\
\hline 2 & 34 & $M$ & 13 & 11 & 21 & 11 & 26 & 28 \\
\hline 12 & 23 & $M$ & 1 & 3 & 1 & 2 & 22 & 33 \\
\hline 14 & 46 & $M$ & 11 & 8 & 8 & 6 & 26 & 34 \\
\hline 22 & 32 & $\mathrm{~F}$ & 13 & 6 & 7 & 7 & 20 & 32 \\
\hline 23 & 25 & $\mathrm{~F}$ & 17 & 7 & 17 & 14 & 24 & 36 \\
\hline 24 & 44 & $M$ & 19 & 17 & 23 & 19 & 30 & 28 \\
\hline 25 & 44 & $M$ & 10 & 2 & 10 & 8 & 20 & 32 \\
\hline 30 & 33 & $M$ & 13 & 13 & 16 & 16 & 30 & 32 \\
\hline 31 & 43 & $M$ & 1 & 1 & 4 & 3 & 20 & 29 \\
\hline 34 & 33 & $M$ & 8 & 11 & 6 & 16 & 22 & 34 \\
\hline 35 & 19 & $M$ & 12 & 11 & 12 & 14 & 32 & 23 \\
\hline 37 & 26 & $M$ & 4 & 5 & 7 & 6 & 20 & 31 \\
\hline
\end{tabular}

\begin{tabular}{|c|c|c|c|c|}
\hline & \multicolumn{2}{|c|}{$\begin{array}{c}\text { Antes de } \\
\text { intervención }\end{array}$} & \multicolumn{2}{|c|}{$\begin{array}{l}\text { Después de } \\
\text { intervención }\end{array}$} \\
\hline & Media & $\mathrm{DE}$ & Media & DE \\
\hline Autoestima* & 24.3 & 4.4 & 31.0 & 3.5 \\
\hline Depresión & 10.2 & 5.7 & 7.9 & 4.8 \\
\hline Ansiedad & 11.0 & 6.9 & 10.2 & 5.6 \\
\hline
\end{tabular}


Figura 1. Prueba Rosemberg de autoestima antes y después de la intervención de Consejería

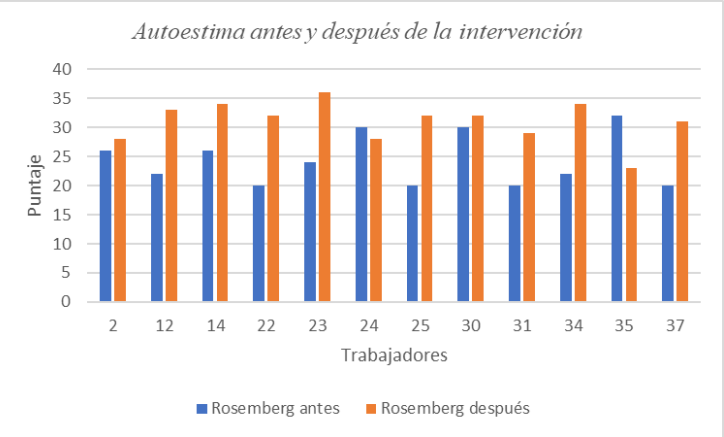

Figura 2. Prueba CESD-10 de depresión antes y después de la intervención de Consejería

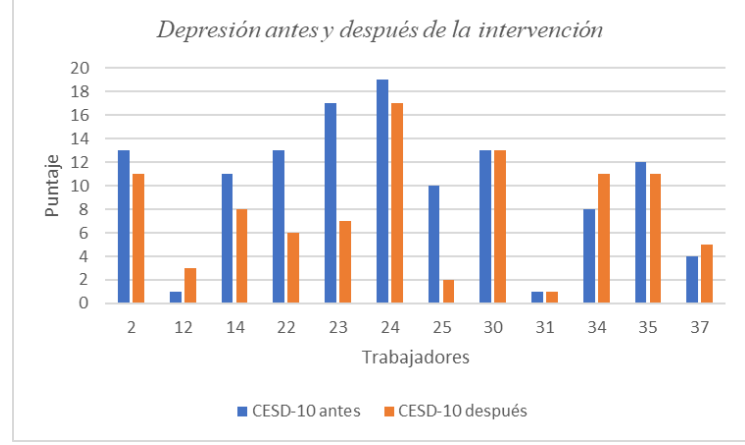

Observamos un aumento de los sentimientos de autovaloración y respeto de sí evaluado como la puntuación de autoestima, antes y después de una intervención en consejería. Con la finalidad de analizar la relación que guardan entre si la autoestima, depresión y ansiedad evaluados como la puntuación obtenida en los diferentes instrumentos utilizados (, se realizó un análisis de correlación para determinar si el aumento en los puntajes de autoestima, se relacionaban con la disminución de los puntajes de depresión y ansiedad (Figura 3). De acuerdo con el análisis de correlación se determinó que el aumento de la puntuación de autoestima se relaciona de forma negativa con los puntajes de depresión y ansiedad ( $\mathrm{r}-0.294$ y $\mathrm{r} \quad-0.171$, respectivamente), pero no es estadísticamente significativo.
Figura 3. Puntuación de sintomatología de autoestima y su relación con la puntuación para depresión y ansiedad.

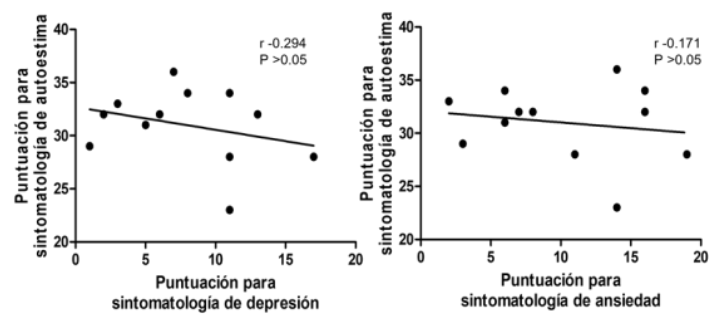

\section{Discusión}

El entorno globalizado y cambiante en el que nos desarrollamos demanda grandes esfuerzos para cumplir con las exigencias de las áreas, personal, familiar, laboral y organizacional, donde la parte emocional psicológica se encuentra comprometida, lo que puede dar como resultado un aumento en el riesgo de trastornos del ánimo como depresión, ansiedad, y como ya mencionamos su interacción con autoestima (Sowislo \& Orth, 2013), así como la conveniencia en términos de productividad de atender a las estrategias de prevención y afrontamiento (Chisholm et al., 2016). La presión organizacional impacta directamente a los trabajadores, si vemos a la salud como un proceso a lo largo de la vida que depende por un lado de la persona $\mathrm{y}$ por otro de las condiciones $\mathrm{y}$ circunstancias que encuentre en su medio, lo que se traduce en bienestar o enfermedad que inciden en la productividad (Durán, 2010).

En este estudio observamos que, aunque los 40 trabajadores iniciales se mostraron entusiasmados por su participación en el estudio solamente lo terminaron a satisfacción 12 personas, por causas muchas veces no atribuibles a ellos mismos sino a eventos laborales. Podemos también incluir la dificultad para dedicar tiempo a estos temas para los que se tiene la percepción de que "no hay tiempo". Se debe competir contra los rápidos cambios tecnológicos, económicos y culturales que nos han llevado a la separación entre los aspectos personal, familiar y laboral.

Al concluir la intervención en consejería los participantes aumentaron la puntuación de autoestima ya que a excepción del trabajador folio 35, 8 participantes obtuvieron puntuaciones que los 


\section{Artículo Original}

clasificaron como autoestima deseable, según Rosemberg. Desconocemos si existen otros factores que ayudaron además de la consejería como por ejemplo la percepción de ser tomados en consideración por su órgano de trabajo. También sería de interés investigar otros aspectos como la resiliencia en cada sujeto. Los participantes también reportaron a sus consejeros sentirse mejor y haber solucionado conflictos en su trabajo y en sus hogares. Lo anterior es consistente con lo reportado por Garay-Sánchez y colaboradores donde el desarrollo de la intervención de consejería demostró cambios significativos en la autoestima evidenciados por las expresiones compartidas con sus consejeros (Gladys, Angel, María, \& Rafael, 2009).

Uno de los resultados esperados consistía en que al concluir la intervención en consejería los participantes aumentaran la puntuación de autoestima y que esto repercutiera en los síntomas de depresión y ansiedad. Sin embargo, no se encontró diferencias significativas para las puntuaciones de depresión y ansiedad, aunque se observa una tendencia en los valores de las pruebas psicológicas. Una posible explicación a esto debido podría ser que, por ejemplo, un participante (folio 34) en lugar de disminuir síntomas, los aumento. Esto pudo deberse a alguna situación que no se contempló en el estudio y de la cual el participante no recibió tratamiento, constituyéndose como una de las limitantes de este trabajo. Por lo cual es necesario más estudios que demuestren el efecto de la consejería en el ámbito laboral y sus resultados a corto, mediano y largo plazo en los síntomas de depresión y ansiedad. Se ha determinado que el estrés laboral puede constituir un desencadenante de la depresión, donde el apoyo de supervisores y una mayor satisfacción en el trabajo pueden disminuir la ocurrencia de estrés laboral y depresión (Gong et al., 2014). Sin embargo, evaluar los niveles de estrés entre los participantes del estudio no fue contemplado, lo cual constituye junto con el tamaño de muestra una de las limitaciones del presente trabajo. Se sugiere que en futuras investigaciones debe realizarse un seguimiento de los participantes que pudieran obtener puntuaciones que los clasifiquen como en riesgo y referirlos al profesional de salud más apropiado, además de evaluar factores como el estrés laboral, que pudieran repercutir en diferentes síntomas relacionados a la salud mental.

\section{Conclusiones}

Se observó un aumento en la puntuación para los sentimientos de autovaloración y respeto de sí mismo, posterior la intervención en consejería. Por lo que se sugiere que las intervenciones de consejería podrían constituir herramientas potenciales para el aumento de la autoestima de trabajadores industriales.

\section{Agradecimientos}

Agradecemos al Ing. Ricardo Jiménez Cataño su contribución invaluable para la realización de este trabajo y su soporte económico para su realización.

Agradecemos a todos los participantes que hicieron posible llevar a cabo este estudio

\section{Bibliografía}

A, T., JB, H., \& RL, B. (2009). Support for the reliability and validity of a six-item state anxiety scale derived from the State-Trait Anxiety Inventory. Journal of nursing measurement, 17(1), 19-28.

https://doi.org/10.1891/1061-3749.17.1.19

Asensio-Cuesta, S., Bresó, A., Saez, C., \& GarcíaGómez, J. M. (2019). Robustness and findings of aweb-based system for depression assessment in a university work context. International Journal of Environmental Research and Public Health, 16(4).

https://doi.org/10.3390/ijerph16040644

Bielecky, A., Chen, C., Ibrahim, S., Beaton, D. E., Mustard, C. A., \& Smith, P. M. (2015). The impact of co-morbid mental and physical disorders on presenteeism. Scandinavian Journal of Work, Environment \& Health, 41(6), 554-564. https://doi.org/10.5271/sjweh.3524

Bower, P., Knowles, S., Coventry, P. A., Rowland, N., \& Group, C. C. M. D. (2011). Counselling for mental health and psychosocial problems in primary care. The Cochrane Database of Systematic Reviews, 2011(9) CD001025. https://doi.org/10.1002/14651858.CD001025.PU B3

Chisholm, D., Sweeny, K., Sheehan, P., Rasmussen, B., Smit, F., Cuijpers, P., \& Saxena, S. (2016). Scaling-up treatment of depression and anxiety: 
A global return on investment analysis. The Lancet Psychiatry, 3(5), 415-424. https://doi.org/10.1016/S2215-0366(16)30024-4

Creswell, C., Violato, M., Fairbanks, H., White, E., Parkinson, M., DClinPsy, ... Cooper, P. J. (2017). Clinical outcomes and cost-effectiveness of brief guided parent-delivered cognitive behavioural therapy and solution-focused brief therapy for treatment of childhood anxiety disorders: a randomised controlled trial. The lancet. Psychiatry, 4(7), 529-539.

https://doi.org/10.1016/S2215-0366(17)30149-9

Evans-Lacko, S., Aguilar-Gaxiola, S., Al-Hamzawi, A., Alonso, J., Benjet, C., Bruffaerts, R., ... Thornicroft, G. (2018). Socio-economic variations in the mental health treatment gap for people with anxiety, mood, and substance use disorders: results from the WHO World Mental Health (WMH) surveys. Psychological Medicine, 48(9), 1560-1571.

https://doi.org/10.1017/S0033291717003336

Gladys, G.-S., Angel, V.-S., María, M.-O., \& Rafael, T.-A. (2009, octubre). Modelo de intervención en consejería grupal como método para reducir el riesgo en la conducta sexual de la mujer con el VIH en tratamiento. Revista Griot, 3(1), 9-22. https://revistas.upr.edu/index.php/griot/article/vi ew/1879/1671

Gong, Y., Han, T., Yin, X., Yang, G., Zhuang, R., Chen, Y., \& Lu, Z. (2014). Prevalence of depressive symptoms and work-related risk factors among nurses in public hospitals in southern China: A cross-sectional study. Scientific Reports 2014 4:1, 4(1), 1-5. https://doi.org/10.1038/srep07109

Hoffmeister, K., Gibbons, A., Schwatka, N., \& Rosecrance, J. (2015). Ergonomics Climate Assessment: A measure of operational performance and employee well-being. Applied Ergonomics, 50, 160-169. https://doi.org/10.1016/j.apergo.2015.03.011

Labaka, A., Goñi-Balentziaga, O., Lebeña, A., \& Pérez-Tejada, J. (2018). Biological Sex Differences in Depression: A Systematic Review. 20(4), 383-392. https://doi.org/10.1177/1099800418776082

Lara-Muñoz, M. del C., Robles-García, R., Orozco, R., Real, T., Chisholm, D., \& Medina-Mora, M. E. (2010). Estudio de costo-efectividad del tratamiento de la depresión en México. Salud Mental, 33(4), 301-308.

Lazo Páez Adrián y Roldan Abellan, Bernie. (2015). Implicaciones del presentismo en la productividad laboral del área de la salud. Medicina legal de Costa Rica, 32(1), 146-153 https://www.scielo.sa.cr/scielo.php?script=sci_ar ttext\&pid=S1409-00152015000100018

Moreno, Y., \& Marrero, R. J. (2015). Optimismo y autoestima como predictores de bienestar personal: diferencias de género. Revista Mexicana de Psicología, 32(1), 27-36.

https://www.redalyc.org/articulo.oa? $\mathrm{id}=2430453$ 63004

Porras-Segovia, A., Rivera, M., Molina, E., LópezChaves, D., Gutiérrez, B., \& Cervilla, J. (2019). Physical exercise and body mass index as correlates of major depressive disorder in community-dwelling adults: Results from the PISMA-ep study. Journal of Affective Disorders, 251, 263-269.

https://doi.org/10.1016/J.JAD.2019.01.050

Rosenberg, M. (2015). Society and the adolescent self-image. Princeton University Pres.

Santomauro, D. F., Mantilla Herrera, A. M., Shadid, J., Zheng, P., Ashbaugh, C., Pigott, D. M., ... Disorders Collaborators, M. (2021). Articles Global prevalence and burden of depressive and anxiety disorders in 204 countries and territories in 2020 due to the COVID-19 pandemic. https://doi.org/10.1016/S0140-6736(21)02143-7

Secretaría del Trabajo y Previsión Social. (2014). Ley Federal del Trabajo.

https://www.gob.mx/stps/documentos/stps-leyfederal-del-trabajo

Sgroi, D. (2010). Does happiness affect productivity? (26 julio 2010). https://voxeu.org/article/does-happiness-affectproductivity 
Shi, Y., Sears, L. E., Coberley, C. R., \& Pope, J. E. (2013). The association between modifiable wellbeing risks and productivity: A longitudinal study in pooled employer sample. Journal of Occupational and Environmental Medicine, 55(4), 353-364.

https://doi.org/10.1097/JOM.0B013E318285192

3

Sowislo, J. F., \& Orth, U. (2013). Does low selfesteem predict depression and anxiety? A metaanalysis of longitudinal studies. Psychological Bulletin, 139(1), 213-240.

https://doi.org/10.1037/a0028931

Sporinova, B., Manns, B., Tonelli, M., Hemmelgarn, B., Macmaster, F., Mitchell, N., ... Quinn, A. (2019). Association of Mental Health Disorders with Health Care Utilization and Costs among Adults with Chronic Disease. JAMA Network Open, 2(8), e199910.

https://doi.org/10.1001/jamanetworkopen.2019.9

910

ST, C., AC, C., \& HH, F. (2006). Factorial structure of a short version of the Center for Epidemiologic Studies Depression Scale. International journal of geriatric psychiatry, 21(4), 333-336.

https://doi.org/10.1002/GPS.1467

Ware, E. B., Mukherjee, B., Sun, Y. V., Diez-Roux, A. V., Kardia, S. L. R., \& Smith, J. A. (2015). Comparative genome-wide association studies of a depressive symptom phenotype in a repeated measures setting by race/ethnicity in the multiethnic study of atherosclerosis. BMC Genetics, 16(1), 118.

https://doi.org/10.1186/s12863-015-0274-0

Wilhelm, K., Martin, G., \& Miranda, C. (2012). Autoestima profesional: competencia mediadora en el marco de la evaluación docente. Revista Latinoamericana de Ciencias Sociales, Niñez y Juventud, 10(1), 339-350.

https://www.redalyc.org/articulo.oa?id=7732398 2020

World Medical Association Declaration of Helsinki: ethical principles for medical research involving human subjects. (2013). JAMA, 310(20), 2191-
2194.

https://doi.org/10.1001/JAMA.2013.281053 